\title{
(1)
}

(7) Volume 4 Issue 2, August 2019

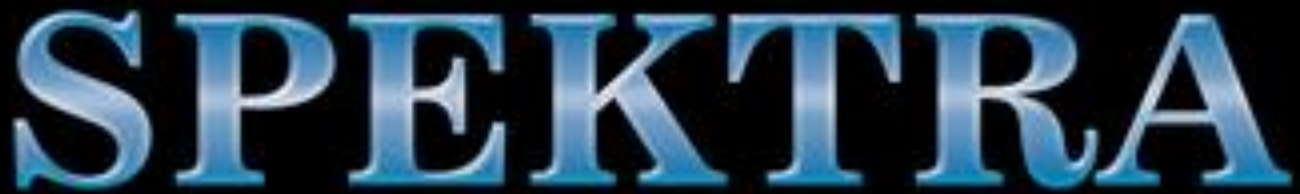

Jurnal Fisika dan Aplikasinya 


\section{SPEKTRA: Jurnal Fisika dan Aplikasinya}

Volume 4 Issue 2, August 2019

DOI: doi.org/10.21009/SPEKTRA.04200

Abstracting and Indexing:

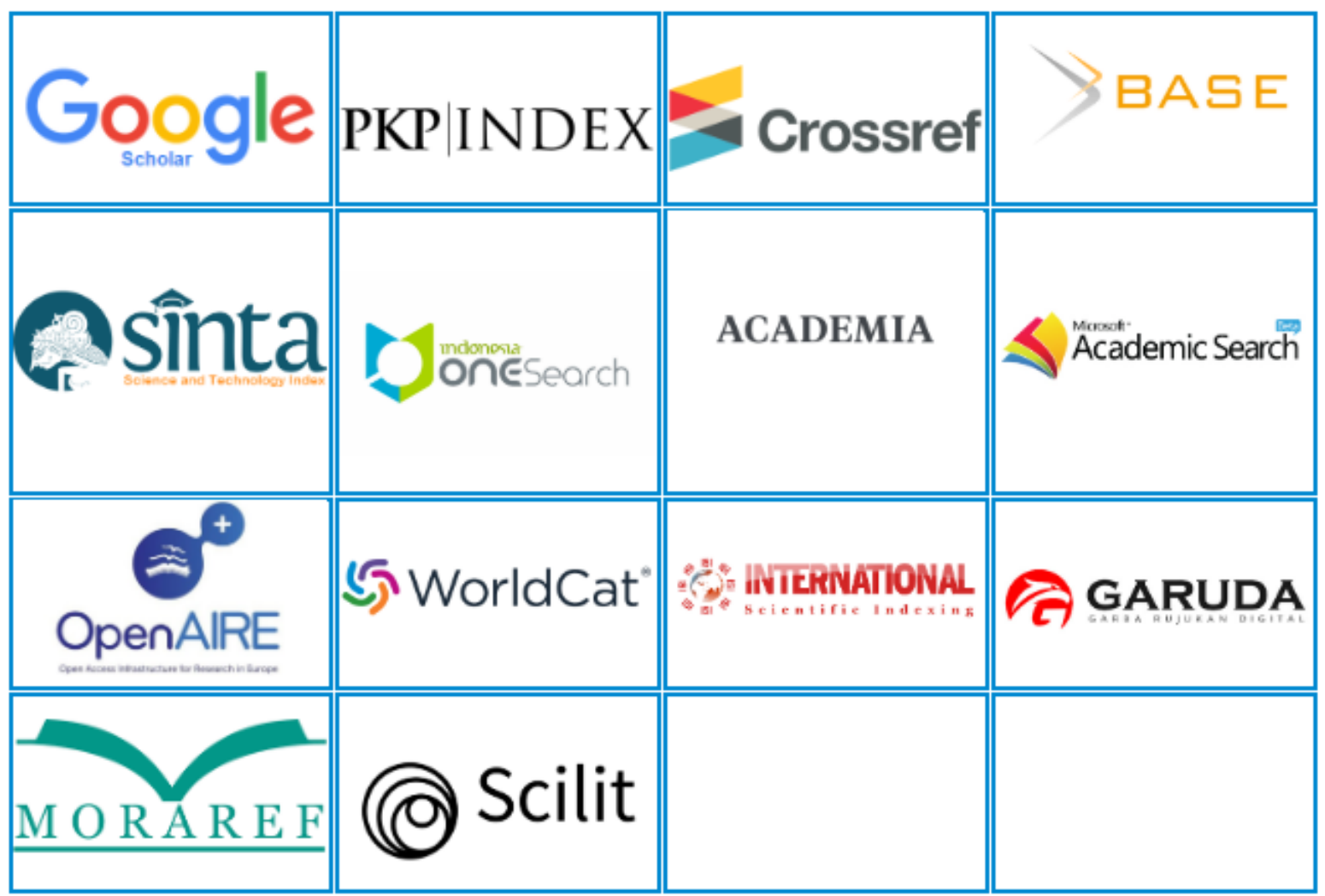


SPEKTRA: Jurnal Fisika dan Aplikasinya

Volume 4 Issue 2, August 2019

THE SPRING VARIATION IN TWO DIMENSIONAL MODELING OF RED BLOOD CELL DEFORMABILITY BASED ON GRANULAR

Ismi Yasifa, Sparisoma Viridi

OPTIMIZATION OF THE USE OF FLY ASH AS AN ADDITIVE PORTLAND COMPOSITE CEMENT (PCC)

Leni Rumiyanti, Listiani Listiani, Tika Damayanti

FLUIDIZATION OF A BED OF SOLID PARTICLES IN A TWO DIMENSIONAL VERTICAL BOX

Aufa Numan Fadhilah Rudiawan, Kurnia Novita Sari, Hirofumi Notsu, Sparisoma Viridi

FRUIT SWEETNESS CHARACTERIZATION USING IMPEDANCE

SPECTROSCOPY METHOD

Rahmondia N Setiadi, Lazuardi Umar

BIOSENSOR SYSTEM DESIGN FOR DISSOLVED OXYGEN MEASUREMENT USING CURRENT MIRROR CIRCUIT

Lazuardi Umar, Rahmondia N Setiadi, Arfianti, Tetty M Linda

MULTI-CHANNEL FRY COUNTER DESIGN USING OPTOCOUPLER SENSOR Lazuardi Umar, Yanuar Hamzah, Rahmondia N Setiadi 


\section{Editor in Chief}

Dr. Widyaningrum Indrasari (Universitas Negeri Jakarta)

\section{Editors}

Prof. Madya Dr. Md. Nizam Abd Rahman (Universiti Teknikal Malaysia Melaka)

Prof. Dr. Agus Setyo Budi, M.Sc. (Universitas Negeri Jakarta)

Dr. Ezza Syuhada Sazali (Universiti Teknologi Malaysia)

Dr. Triati Dewi Kencana Wungu (Institut Teknologi Bandung)

Dr. Iwan Sugihartono, M.Si. (Universitas Negeri Jakarta)

Dr. Esmar Budi, M.T. (Universitas Negeri Jakarta)

Dr. Irzaman, M.Si. (Institut Pertanian Bogor)

Dr. -Ing. Rahmondia Nanda Setiadi, M.Si. (Universitas Riau)

Dr. Yulkifli, M.Si. (Universitas Negeri Padang)

Dr. Idha Royani, M.Si. (Universitas Sriwijaya)

Dr. Masturi (Universitas Negeri Semarang)

Dr. Rahadi Wirawan, M.Si. (Universitas Mataram)

Dr. Eleonora Agustine, M.Si. (Universitas Padjadjaran)

Dr. Lilik Hasanah (Universitas Pendidikan Indonesia)

Dr. Teguh Budi Prayitno, M.Si. (Universitas Negeri Jakarta)

Yanurita Dwi Hapsari, M.Sc. (Institut Teknologi Sepuluh Nopember)

\section{Editorial Office}

Program Studi Fisika Fakultas MIPA

Kampus A Universitas Negeri Jakarta

Gedung K.H. Hasyim Asyari Lt.10

Jalan Rawamangun Muka No.1

Rawamangun-Pulogadung Jakarta Timur, 13220 


\section{Reviewers (Mitra Bebestari)}

Prof. Yusaku Fujii (Gunma University)

Prof. Hsiang-Lin Liu (National Taiwan Normal University)

Prof. Md. Rahim Sahar (Universiti Teknologi Malaysia)

Dr. Ramli, M.Si. (Universitas Negeri Padang)

Dr. Fiber Monado (Universitas Sriwijaya)

Dr. Zaroh Irayani (Universitas Jenderal Sudirman)

Dr. Siti Zulaikah, M.Si. (Universitas Negeri Malang)

Dr. Sparisoma Viridi (Institut Teknologi Bandung)

Dr.rer.nat. Bambang Heru Iswanto, M.Si. (Universitas Negeri Jakarta)

Dr. Sunaryo, M.Si. (Universitas Negeri Jakarta)

Dr. Mangasi Alion Marpaung, M.Si. (Universitas Negeri Jakarta)

Dr. Erfan Handoko, M.Si. (Universitas Negeri Jakarta)

Dr. Anggara Budi Susila, M.Si. (Universitas Negeri Jakarta)

Dr. Mutia Delina, M.Si. (Universits Negeri Jakarta)

Dr. Trismidianto, M.Si. (Pusat Sains dan Teknologi Atmosfer, LAPAN)

Dr. Lusi Safriani, M.Si. (Universitas Padjadjaran)

Dr. Agus Supriyanto, M.Si. (Universitas Negeri Sebelas Maret)

Dr. Umiatin, M.Si. (Universitas Negeri Jakarta)

Taufiq Hidayah, M.Si. (Pusat Meteorologi BMKG)

Prihatin Oktivasari, M.Si. (Politeknik Negeri Jakarta)

Mera Kartika Delimayanti, M.T. (Politeknik Negeri Jakarta)

Dewi Muliyati, M.Si.,M.Sc. (Universitas Negeri Jakarta)

Riser Fahdiran, M.Si. (Universitas Negeri Jakarta) 


\section{EDITORIAL FOREWORD}

SPEKTRA: Jurnal Fisika dan Aplikasinya is dedicated to all physics practitioners. The coverage of SPEKTRA includes: Instrumentation and Computational Physics, Material Physics, Medical Physics and Biophysics, Astrophysics, Theoretical Physics, Particle and Nuclear Physics, Environment Physics, Renewable Energy, and other fields related to the application of physics.

SPEKTRA Volume 4 issue 2 contains 6 papers: 1) The Spring Variation in Two Dimensional Modeling Of Red Blood Cell Deformability Based on Granular; 2) Optimization of The Use of Fly Ash Is An Additive Portland Composite Cement (PCC); 3) Fluidization of A Bed of Solid Particles In A Two Dimensional Vertical Box; 4) Fruit Sweetness Characterization Using Impedance Spectroscopy Method; 5) Biosensor System Design for Dissolved Oxygen Measurement Using Current Mirror Circuit; 6) Multi-Channel Fry Counter Design Using Optocoupler Sensor.

Hopefully, SPEKTRA can be a reference for readers and researchers in developing physics and its application.

Jakarta, 31 August 2019

Editor-in-Chief,

Widyaningrum Indrasari 


\section{CONTENTS}

TITLE AND AUTHOR

THE SPRING VARIATION IN TWO DIMENSIONAL MODELING OF RED BLOOD CELL DEFORMABILITY BASED ON GRANULAR

Ismi Yasifa, Sparisoma Viridi

OPTIMIZATION OF THE USE OF FLY ASH AS AN ADDITIVE PORTLAND COMPOSITE CEMENT (PCC)

Leni Rumiyanti, Listiani Listiani, Tika Damayanti

FLUIDIZATION OF A BED OF SOLID PARTICLES IN A TWO DIMENSIONAL VERTICAL BOX

Aufa Numan Fadhilah Rudiawan, Kurnia Novita Sari, Hirofumi Notsu, Sparisoma Viridi

FRUIT SWEETNESS CHARACTERIZATION USING IMPEDANCE SPECTROSCOPY METHOD

Rahmondia N Setiadi, Lazuardi Umar

BIOSENSOR SYSTEM DESIGN FOR DISSOLVED OXYGEN

MEASUREMENT USING CURRENT MIRROR CIRCUIT

Lazuardi Umar, Rahmondia N Setiadi, Arfianti, Tetty M Linda

MULTI-CHANNEL FRY COUNTER DESIGN USING OPTOCOUPLER SENSOR

Lazuardi Umar, Yanuar Hamzah, Rahmondia N Setiadi
PAGES

$51-60$

$61-72$

$73-80$

$81-88$

$89-96$

$97-104$ 
